\title{
Development and physico-chemical characterization of virgin coconut oil-in-water emulsion using polymerized whey protein as emulsifier for Vitamin A delivery
}

\author{
Erin Jasse Tanglao ${ }^{1}$, Arun Bryan Nanda Kumar ${ }^{1}$, Ronald Ryan Noriega ${ }^{1}$, Mark Emile Punzalan ${ }^{1}$, and Philipina Marcelo ${ }^{1}$ \\ ${ }^{1}$ Chemical Engineering Department, Faculty of Engineering, University of Santo Tomas, España Boulervard 1015 Manila, \\ Philippines
}

\begin{abstract}
Emulsion systems have become an integral part of food manufacturing, mainly as delivery vehicles of sensitive lipophilic nutrients i.e. Vitamin A. Thus, it is crucial to develop emulsions stable enough to protect these nutrients. In this study, virgin coconut oil (VCO)-in-water emulsion was prepared with polymerized whey protein as emulsifier to encapsulate Vitamin A. The VCO droplets were dispersed in water phase, and Vitamin A was dissolved in oil phase. The study aimed at developing VCO-in-water emulsion to encapsulate and protect Vitamin A, in the form of retinyl acetate, and determining physicochemical characteristics of the emulsion. In vitro analysis was conducted to determine the stability of the emulsion in encapsulating Vitamin A. Three emulsions were prepared at different homogenization speed: $720,846.7$ and $955.8 \mathrm{rpm}$. The emulsion made with speed setting of $846.7 \mathrm{rpm}$ gave the best visual characteristics, comparable with dairy butter. Thermal analyses using differential scanning calorimeter showed that the emulsion increased the energy requirement to degrade Vitamin A at simulated stomach $\mathrm{pH}$. Also, microscopy results show the emulsion has an average particle diameter of approximately $10 \mu \mathrm{m}$, which remained stable at acidic environment of simulated digestion. Therefore, the emulsion is thermodynamically stable and shows minimal coalescence.
\end{abstract}

\section{Introduction}

Nutrients play an essential role in the proper development of the human body. However, much of the viable nutrients in food are lost during food processing. This has led to encapsulation being the most employed method in food processing to conserve, protect and deliver the needed nutrients to the body [1]. Encapsulation is used to expand the bioactive molecule delivery in foods and can also alter the physical features of the original substance without altering the quality of the substance. As such, different methods in which nutrients can be delivered through encapsulation delivery systems in the micro- and nano-scales [2]. Currently, emulsions are used as delivery vehicles within the food industry, making it an integral part of food manufacturing [3].

Emulsions have great potential in encapsulating high concentration of oil-soluble nutraceuticals or bioactive food supplements into wide range of food. The delivery of any bioactive compound to various sites within the body is, however, directly affected by the oil droplet size of the emulsion [4-5]. Careful selection of an emulsifier enables control over interfacial properties, thickness, rheology, and response to environmental conditions [6]. In this study polymerized whey protein isolate will act as the emulsifier as an unfolded globular whey protein leads to opening of reactive sites of the protein where hydrophobic and hydrophilic groups may be arranged for good aggregation results [7-9].

For this study, the o/w emulsion will act as delivery vehicles with the whey protein polymer coating the oil droplets with Vitamin A.

Generally, the research aims to develop an oil-inwater emulsion that can be utilized as a medium for nutrient delivery. Specifically, the research aims to: develop a whey protein polymer-emulsified-VCO-inwater emulsion with encapsulated Vitamin A in the oil phase; characterize the resulting emulsion in terms of its physico-chemical properties in relation to its suitability as a Vitamin A delivery system; and evaluate the encapsulated Vitamin A in the emulsion in vitro.

The o/w emulsion will improve the distribution and permeation on application.

\section{Material and methods}

\subsection{Materials and emulsion creation methodology}

Whey protein isolate (WPI) used was HilmarTM 9400 acid stable whey protein isolate. WPI was obtained from CTC Far East Philippines Incorporated, the local

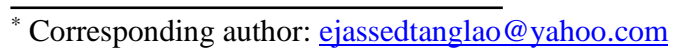


distributor of Hilmar Ingredients, based in Mandaluyong City, Philippines. The virgin coconut oil (VCO) used in the study was a commercial brand named Carica, purchased from supermarkets. Vitamin A used in this study was in retinyl acetate form, which is the predominant form of the vitamin. The vitamin was from SentroTek Incorporated, which is also located in Mandaluyong City, Philippines. Stability of the emulsion is highly desired [8-9], thus the methodology for developing the emulsion is based on the study of Macaraeg et. al (2010) [7].

\subsection{Preparation of whey protein isolate solution}

Ultrapure water obtained from the University of Santo Tomas Thomas Aquinas Research Center (UST TARC), was used as the solvent for WPI powder to form the WPI solution with $15 \%(\mathrm{w} / \mathrm{w})$ concentration in a $250-\mathrm{mL}$ Pyrex beaker. The WPI powder was dissolved using Corning stirrer/hot plate set-up for 90 minutes at a controlled speed to avoid vortex formation. The WPI solution made was then sealed with a plastic paraffin film and left overnight in a refrigerator at approximately $4^{\circ} \mathrm{C}$ to equilibrate.

\subsection{Thermal polymerization}

Polymerization of WPI solution to form WPI polymer was done through a hot water bath. The WPI solution was subjected to constant uniform stirring to ensure equal distribution of heat. Heating temperature of the solution was maintained at $70 \pm 1{ }^{\circ} \mathrm{C}$. The solution was heated for 25 minutes as developed in previous experimental study [7]. The temperature of the solution was recorded at 2-minute intervals. The heated WPI polymer was then cooled to room temperature prior to being used as an emulsifier of VCO.

\subsection{Incorporation of retinyl acetate in VCO}

About $0.70 \mathrm{mg}$ of vitamin $\mathrm{A}$ in the form of retinyl acetate was dispersed in $90 \mathrm{~mL}$ of VCO using the Corning hot plate/magnetic stirrer. $\mathrm{VCO}$ with retinyl acetate was continuously stirred to avoid settling of the vitamin A. Approximately $10 \mathrm{~mL}$ of $\mathrm{VCO}$ was used to wash the beaker containing the retinyl acetate prior to emulsification.

\subsection{Preparation of whey protein polymer- emulsified-VCO-in-water emulsion and determination of the homogenization rate}

An emulsion with oil concentration of $50 \%(\mathrm{v} / \mathrm{v})$ was prepared using VCO and polymerized whey protein isolate (PWPI). Emulsion was prepared by the slow addition of $5 \mathrm{~mL}$ of $\mathrm{VCO}$ every 2 minutes. The hand mixer used was a National Hand mixer with 5 speed settings. To determine the best homogenization rate, three emulsions were prepared using the three different speeds of the hand mixer. These emulsions were then tested for their visual appearance and texture.

\subsection{Obtaining the physical properties of the emulsion}

Viscosity of the emulsions was determined using a constant shear Brookfield viscometer. Density of the emulsion was determined using a standard $25 \mathrm{~mL}$ Pycnometer. The measurements were done in triplicates. Viscosity was plotted versus the shear rate to obtain the viscosity profile of the emulsion. The density was compared to the density of common butter.

\subsection{In vitro analysis of VCO-PWPI emulsion with Vitamin A}

Approximately $100 \mathrm{~g}$ of the prepared whey proteinpolymer-emulsified-VCO-in-water emulsion was mixed with $100 \mathrm{~mL} \mathrm{HCl}$ with a $\mathrm{pH}$ of 1.67 . This was done in order to stimulate the activity happening in the stomach, where the food is mixed with enteroendocrine $G$ cells which secrete gastrin hormone into blood which stimulates an acidic environment, with a $\mathrm{pH}$ ranging from 1-3 [8]. To simulate extreme digestion conditions, the emulsion and acid were mixed in a beaker, stirred, and transferred into a $200 \mathrm{~mL}$ amber bottle and stored for 6-hours. Differential Scanning Calorimetry (DSC) analysis was then performed on simulated digested and undigested samples.

\subsection{Differential scanning calorimetry}

The thermogram of vitamin A was obtained by weighing $7.7 \mathrm{mg}$ of vitamin A and loading it into a Q200 DSC (TA Instruments). The temperature range for the vitamin $\mathrm{A}$ is $-100^{\circ} \mathrm{C}$ to $250{ }^{\circ} \mathrm{C}$. The fresh emulsion and emulsion in $\mathrm{HCl}$, with the $\mathrm{pH}$ of 1.67 , were weighed $12.5 \mathrm{mg}$ and $31.1 \mathrm{mg}$, respectively, and loaded into a Q200 DSC (TA Instruments). The temperature range for both samples is $-100^{\circ} \mathrm{C}$ to $250{ }^{\circ} \mathrm{C}$.

\subsection{Transmitted light frame microscopy}

The microstructure of the $50 \%(\mathrm{v} / \mathrm{v})$ VCO-in-water emulsion was determined using an Olympus IX51. Peasized amount of emulsion sample was spread onto a glass slide and covered. The magnification used is $40 \mathrm{x}$.

\section{Results and discussion}

\subsection{Emulsion characteristics}

Three samples of whey protein polymer-emulsified VCO-in-water emulsions were prepared, each at a different hand mixer speed setting of 720, 846.7 and $955.8 \mathrm{rpm}$ or E1, E2, and E3, respectively. All emulsions were prepared using $50 \%(\mathrm{v} / \mathrm{v})$ oil concentrations. Emulsion mixing speed selection was solely based on 
visual appearance and texture. E1 and E3 contained lumps of polymerized whey protein and air bubbles, respectively, prompting E1 and E3 to be deemed unfit to be used in the study. E2 had a smooth and creamy, peanut-butter like texture which is desirable for the study.

\subsection{Physical properties}

Density and viscosity of the emulsion were measured and recorded because this will help the researchers in identifying the appropriate mixing speed setting for the emulsion. The density helped the researchers obtain the desired texture of the emulsion. It was measured in triplicates as shown in Table 1.

Table 1. Density of the emulsion

\begin{tabular}{|c|c|}
\hline Mass of Sample $(\mathrm{g})$ & Density $(\mathrm{g} / \mathrm{mL})$ \\
\hline 11.7546 & 1.17546 \\
\hline 8.7185 & 0.87185 \\
\hline 8.6355 & 0.86355 \\
\hline
\end{tabular}

The average density is $0.9703 \pm 0.1777 \mathrm{~g} \mathrm{~mL}^{-1}$, which when compared to $0.9602 \mathrm{~g} \mathrm{~mL}-1$ from the past study of Macaraeg et al. (2010) resulted to $1.05 \%$ difference. Moreover, as the researchers aim to have texture that is easily spreadable, the VCO-in-water emulsion was also compared to common butter, which has a density of $0.911 \mathrm{~g} \mathrm{~mL}^{-1}$ [11]. The per cent difference in density was $6.51 \%$. On the other hand, viscosity, as shown in Table 2, helped the researchers to observe the emulsifying capacity of the whey protein polymer in the VCO-inwater emulsion.

Table 2. Viscosity of the emulsion.

\begin{tabular}{|c|c|c|}
\hline $\begin{array}{c}\text { Shear Rate } \\
(1 / \mathrm{s})\end{array}$ & Apparent Viscosity (Pa-s) & $\begin{array}{c}\text { Shear Stress } \\
(\mathrm{Pa})\end{array}$ \\
\hline 0.005 & 100 & 0.5 \\
\hline 0.01 & 60 & 0.6 \\
\hline 0.025 & 40 & 1 \\
\hline 0.1 & 21 & 2.1 \\
\hline 0.2 & 12 & 2.4 \\
\hline
\end{tabular}

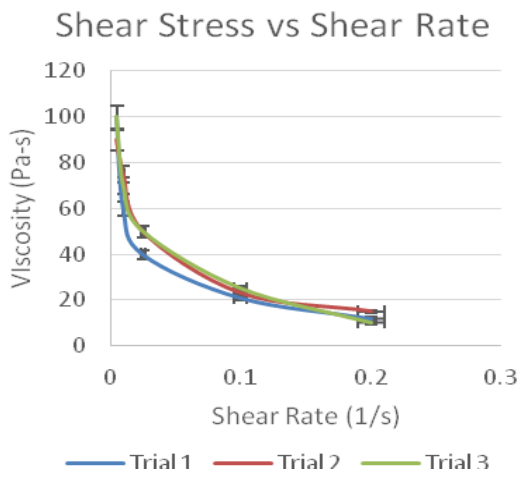

Fig. 1. Viscosity versus shear rate of the emulsion.

The emulsion prepared exhibited the theoretical behavior of a pseudoplastic fluid as shown in Fig. 1 . This observed behavior of the emulsions in this study is consistent with generally accepted behavior of emulsions. According to Campanella et al. [11], most emulsions with oil concentrations ranging from $10 \%$ to $70 \%$ exhibit Non-Newtonian behavior.

\subsection{Stability of the developed emulsion}

It is shown that a VCO-in-water emulsion created using $70 \mathrm{C} \pm 1 \mathrm{C}$ as the polymerization temperature with a heating time of 25 minutes gives satisfactory results in terms of emulsion stability. Fig. 2 shows the percentage of VCO emulsified by the polymerized whey protein and its stability when subjected to refrigeration [7].

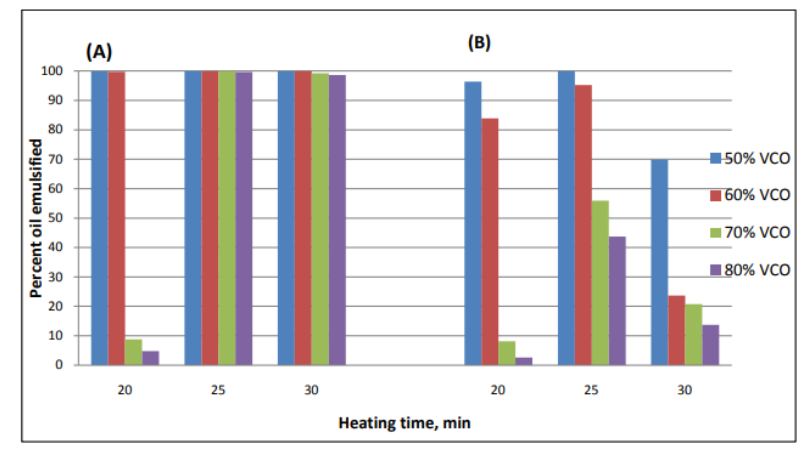

Fig. 2. Percentage of oil emulsified from (A) freshly prepared emulsions and (B) refrigerated emulsions at $4^{\circ} \mathrm{C}$ for 1 hour. Both are at various oil concentrations [7].

\subsection{Differential scanning calorimetry}

Three samples were subjected to DSC test: pure vitamin A, VCO-in-water emulsion encapsulating vitamin A, and emulsion encapsulating vitamin A subjected to simulated stomach $\mathrm{pH}$. This was done to observe their thermal stability as well as the energy required to disrupt the system. The thermogram of Retinyl Acetate is shown in Fig 3. Thus, encapsulation of vitamin $\mathrm{A}$ is needed to make it more stable under extreme conditions and to be efficiently absorbed by the body.

Comparing Figs. 4 and 5, only the second peak from the vitamin A thermogram appeared. These show that the peaks occurred at almost the same temperature but differ in terms of the heat flow. This difference in heat flow denotes a change in the heat requirement of the system. The peak around $134^{\circ} \mathrm{C}$ in the thermogram of the emulsion indicates that it needed higher energy to appear compared to the peak in the vitamin A thermogram.

According to Wagner (2017), exothermic chemical reactions and vaporization only appear during the first heating cycle in DSC analysis. The first peaks in the thermograms of the emulsions indicate the vaporization of water. This is also strengthened by the fact that the first peak of Fig. 4 has a larger area than the first peak of Fig. 5 indicating that a higher amount of water is present in the sample as it was mixed with $\mathrm{HCl}$ solution to simulate digestion. 


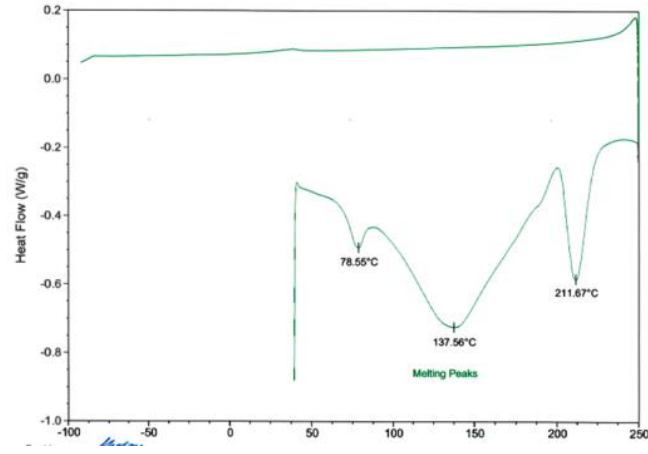

Fig. 3. Thermogram of dry vitamin A

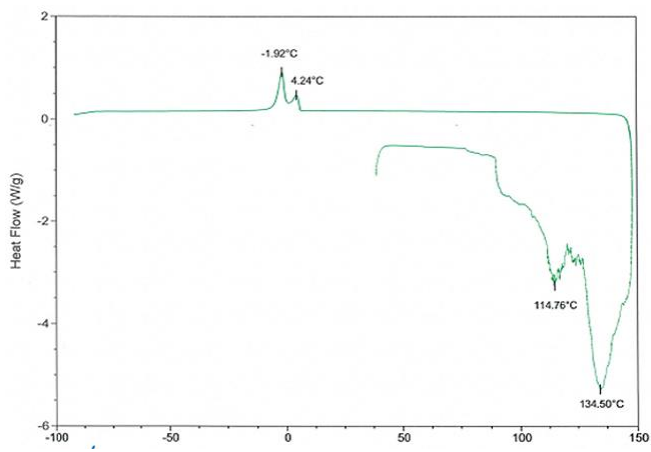

Fig. 4. Thermogram of the VCO-WPI emulsion with Vitamin A.

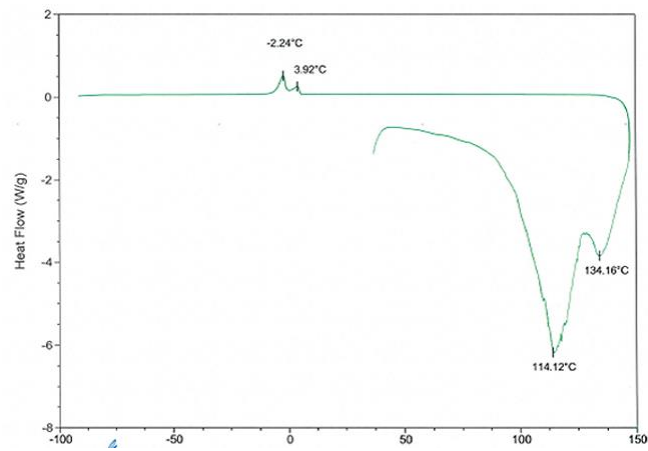

Fig. 5. Thermogram of the VCO-WPI with vitamin $\mathrm{A}$ in $\mathrm{HCl}$

\subsection{Transmitted light frame microscopy}

Four samples of the VCO-WPI emulsion were observed under the microscope using Transmitted Light Frame Microscopy model Olympus IX51. Three of the said samples were mixed with the acid, each with a specific time of mixing with the acid: 30 minutes, 1 hour and 1.5 hrs. The digestion time of whey protein in the stomach is around 1.5 hours [12]. The resulting microstructure of the emulsion showed satisfactory stability. As shown in Fig. 6, the whey protein polymer coats the oil droplets. This indicates that the oil droplets kept their round shape and are closely-packed without coalescing.

Table 3. Mean and median droplet size for the four emulsions

\begin{tabular}{|c|c|c|}
\hline Time & Mean & Median \\
\hline 0 & 10.6501 & 10.6850 \\
\hline 30 minutes & 10.3856 & 10.0995 \\
\hline 1 hour & 10.7399 & 10.6405 \\
\hline 1.5 hours & 1.7594 & 10.4595 \\
\hline
\end{tabular}

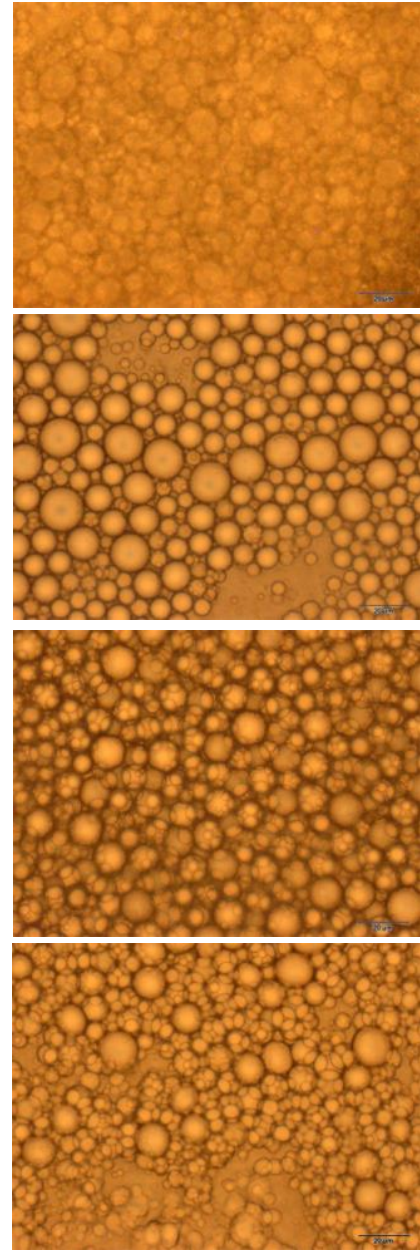

Fig. 6. Images of the emulsions obtained. Fresh Emulsion (upper-left), after 30 minutes (upper-right), 1 hour (lower-left), 1.5 hours (lower-right). Images at 40x magnification.

The mean and median droplet sizes for these images are shown in Table 3 while Figs. 7-10 show the droplet size distribution for each sample. This was done to show that the droplet size frequency is concentrated at a certain size. This was done by randomly selecting 100 droplets per image and measuring the diameters using the $20 \mu \mathrm{m}$ scale (as shown in the image). The measurements were done using ImageJ and the data gathered were further interpreted using Microsoft Excel. As previously stated, uniformity of droplet size is important because it greatly affects the stability of the emulsion. Therefore, for micro-emulsions smaller droplet size is desired and must have a droplet size less than or equal to $10 \mu \mathrm{m}$ [10]. 


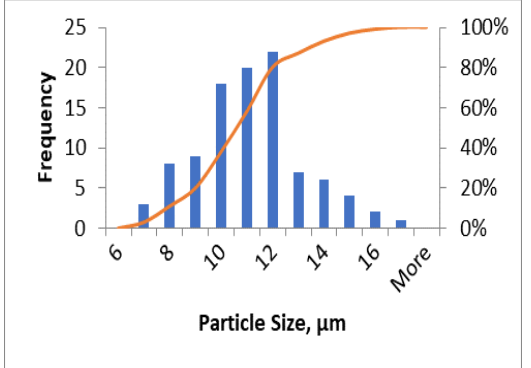

Fig. 7. Droplet distribution for the fresh emulsion

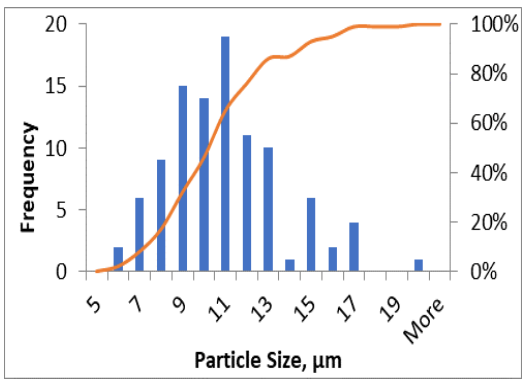

Fig. 8. Droplet Distribution after 30 minutes

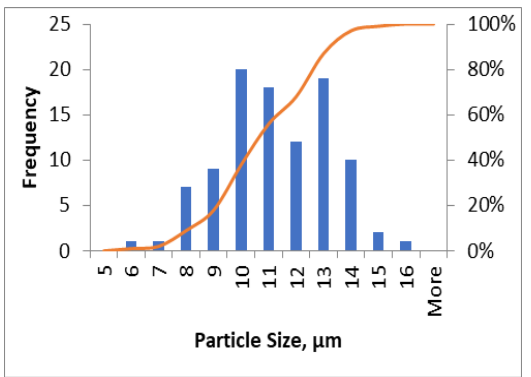

Fig. 9. Droplet Distribution after 1 hour

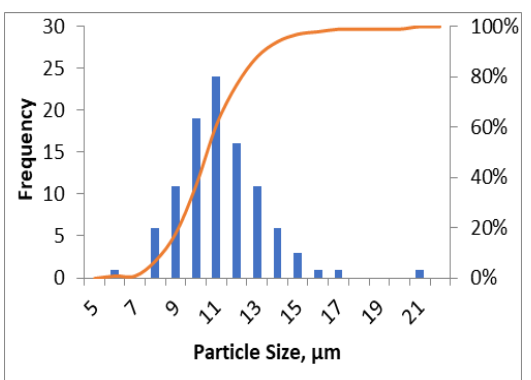

Fig. 10. Droplet Distribution after 1.5 hours

\section{Conclusion}

The study demonstrates the development of a VCO-inwater emulsion with polymerized whey protein isolate as emulsifier to encapsulate Vitamin A. The emulsion preparation method developed by Macaraeg, et al, (2010) [7] was modified to encapsulate Vitamin A through the emulsion. Results displayed success in characterizing the emulsion in terms of physico-chemical characteristics. In addition, thermal analysis and microscopical analysis displayed the capability of the emulsion to protect of Vitamin A in vitro.

The DSC thermogram, used to characterize the energy requirement of the breakdown of the emulsion, proved whey protein polymer as emulsifier to be thermally stable for the protection of Vitamin A. Microscopical analysis of the emulsion supports the DSC thermogram in displaying Vitamin A protection in simulated digestion. Extraneous particle size and coalescence was avoided due to the emulsifying capabilities of the polymerized whey protein and the mixing speed. The emulsion was discovered to have droplet sizes in the range of 5-20 $\mu \mathrm{m}$. Therefore, the developed emulsion was stable enough to protect the Vitamin A, from high temperatures, and low $\mathrm{pH}$.

It is recommended for the study to be conducted using other sensitive micronutrients in order to add more nutritional value to the emulsion. Using a homogenizer is also recommended for a controlled mixing and further breakdown of oil droplets as stability of the emulsion may be increased with a smaller droplet size. Lastly, it is recommended for a sensory evaluation and organoleptic testing to be performed to further modify the emulsion to become consumer friendly and marketable to the public as an alternative to butter.

The authors would like to express their deepest gratitude to our adviser and friends who have been thoroughly supportive in this endeavor. We would also like to thank CTC Group Philippines for the aid in providing whey protein isolate and SentroTek Inc. for providing retinyl acetate for the study.

\section{References}

[1] Smith, M. E., Coffin, A. B., Miller, D. L., \& Popper, A. N. (2006). Anatomical and functional recovery of the goldfish (Carassius auratus) ear following noise exposure. Journal of Experimental Biology, 209(21), 4193-4202. https://doi.org/10.1242/jeb.02490

[2] Karunaratne, D. N., Siriwardhana, D., Ariyarathana, I. R., Rajakararuna, R. M. P. I., Banu, F. T., \& Karunaratne, V. (2017). Nutrient Delivery Through Nanoencapsulation.

https://doi.org/https://doi.org/10.1016/B978-0-12804304-2.00017-2

[3] Lakkis, J. (2016). Encapsulation and Controlled Release Technologies in Food Systems (Second). Barcelona: John Wiley \& Sons, Ltd.

[4] Ghorani, B., \& Tucker, N. (2015). Fundamentals of electrospinning as a novel delivery vehicle for bioactive compounds in food nanotechnology. Food Hydrocolloids, $\quad 51, \quad 227-240$. https://doi.org/10.1016/j.foodhyd.2015.05.024

[5] McClements, D. J., \& Jafari, S. M. (2018). Improving emulsion formation, stability and performance using mixed emulsifiers: A review. Advances in Colloid and Interface Science, 251, 55-79. https://doi.org/10.1016/j.cis.2017.12.001

[6] Euston, S. R., Finnigan, S. R., \& Hirst, R. L. (2002). Kinetics of droplet aggregation in heated whey protein-stabilized emulsions: Effect of polysaccharides. Food Hydrocolloids, 16(5), 499505. https://doi.org/10.1016/S0268-

[7] Macaraeg, G., Sebastian, L.J., Tan, M.K., \& Marcelo, P. (2010). Characterization of virgin 
coconut oil emulsions using polymerized whey protein in aqueous solution

[8] Gupta, C., Arora, S., Syama, M. A., \& Sharma, A. (2017). Preparation of milk protein-vitamin A complexes and their evaluation for vitamin A binding ability. Food Chemistry, 237, 141-149. https://doi.org/10.1016/j.foodchem.2017.05.106

[9] Campanella, O., Dorward, N., \& Singh, H. (1995). A study of the rheological properties of concentrated food emulsions. Journal of Food Engineering, 25(3), 427-440.

[10] Eitel, J. (2017). How Fast Do You Digest Whey
Protein?
Retrieved
from

https://www.livestrong.com /article/520142-howfast-do-you-digest-whey-protein/\#

[11] Charrondiere, U. R., Haytowitza, D., Stadlmayr, B. (2012). FAO/INFOODS Density Database. https://www.fao.org/docrep/017/ap815e/ap815e.pdf 